\title{
Editorial
}

\section{Transgender Voice: The Challenges}

Transgender voice is an evolving field in laryngology. Across the globe and even in India, more number of clients is increasingly approaching the laryngologist, seeking solution to this problem. It poses a great challenge to the clinician, as no single procedure has been able to stand the test of time till date.

Hormone therapy can effectively lower the pitch in female to male transgender subjects. It fails to bring about any improvement in voice quality in male to female transgenders, as feminizing hormones have no direct effect on vocal folds.

In male to female transgender population, acquiring a gender appropriate voice plays a key

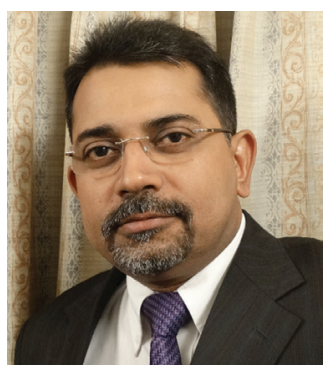
role in their acceptance in the newly assigned gender. Speech and language pathologists can train them to produce high-pitched voice in natural "feminine intonation." Voice therapy usually results in production of desirable highpitched voice by voluntary contraction of intrinsic and extrinsic muscles. However, some individuals face extreme fatigue with ongoing voice usage. Second, at times, their original male voice may show up momentarily, especially during and after nonverbal act like sneezing and coughing.

Numerous surgical techniques have therefore evolved to address this issue. One of the most popular and age-old pitch raising surgeries is cricothyroid approximation, where the thyroid and cricoid cartilage are sutured together mimicking a permanent contraction of cricothyroid muscle. Though, this technique is simple and safe, many patients experience an unnaturally high pitched and harsh voice and in some, the initially raised pitch drops back to baseline over a few months.

This prompted many surgeons from different parts of the world to formulate newer surgical techniques. Laserassisted voice adjustment uses the principle of thinning and tightening the vocal folds with or without anterior commissure webbing. Drastic shortening of vocal folds along with retrodisplacement of anterior commissure has also been reported with good outcome. Shortening of true and false vocal folds in conjunction with thyrohyoid approximation is also in practice, which alters the resonance in addition to elevation of pitch, without a strained voice.

Transition from masculine to feminine voice not only translates to elevation of pitch, but also demands alteration of resonance, timber, and the style of speaking. Any surgical procedure, which enables shortening and thinning of vocal folds with slight laryngeal elevation, with or without minimal volume reduction of larynx and pharynx is expected to bring about superior outcome. Voice therapy before and after surgery is mandatory to finally tune the voice to match the desire of each individual. We must look forward to the future and wait for a new horizon when stem cell therapy may be able to "feminize" the larynx; this may preclude many extensive surgical procedures in the days to come.

Amitabha Roychoudhury Editor-in-Chief International Journal of Phonosurgery and Laryngology Professor and Head Department of ENT and Head-Neck Surgery Vivekananda Institute of Medical Sciences Ramakrishna Mission Seva Pratishthan Kolkata, West Bengal, India E-mail: aroychoudhury@yahoo.com 